typeset using JPSJ.sty < ver.1.0b>

\title{
Critical Temperature of Ferromagnetic Transition in Three-Dimensional Double-Exchange Models
}

\author{
Yukitoshi Motome and Nobuo Furukawa ${ }^{1}$ \\ Institute of Materials Science, University of Tsukuba, Tsukuba, Ibaraki 305-0006 \\ ${ }^{1}$ Department of Physics, Aoyama Gakuin University, Setagaya, Tokyo 157-8572
}

(Received )

\begin{abstract}
Ferromagnetic transition in three-dimensional double-exchange models is studied by the Monte Carlo method. Critical temperature $T_{\mathrm{c}}$ is precisely determined by finite-size scaling analysis. Strong spin fluctuations in this itinerant system significantly reduce $T_{\mathrm{c}}$ from mean-field estimates. By choosing appropriate parameters, obtained values of $T_{\mathrm{c}}$ quantitatively agree with experiments for the ferromagnetic metal regime of $(\mathrm{La}, \mathrm{Sr}) \mathrm{MnO}_{3}$, which is a typical perovskite manganite showing colossal magnetoresistance. This indicates that the double-exchange mechanism alone is sufficient to explain $T_{\mathrm{c}}$ in this material. Critical exponents are also discussed.
\end{abstract}

KEYWORDS: double-exchange model, colossal magnetoresistance manganites, ferromagnetic transition, critical temperature, finite-size scaling, Monte Carlo method

Colossal magnetoresistance near the ferromagnetic transition has attracted much attention to the strong interplay between transport and magnetism in perovskite manganese oxides. 2. This feature has been attributed to the double-exchange (DE) mechanism 3 in the Hamiltonian

$$
\mathcal{H}=-\sum_{i j} \sum_{\sigma=\uparrow, \downarrow} t_{i j}\left(c_{i \sigma}^{\dagger} c_{j \sigma}+\text { h.c. }\right)-J_{\mathrm{H}} \sum_{i} \boldsymbol{\sigma}_{i} \cdot \boldsymbol{S}_{i},
$$

where $c_{i \sigma}\left(c_{i \sigma}^{\dagger}\right)$ annihilates (creates) a $\sigma$-spin electron at site $i$, and $J_{\mathrm{H}}$ is the Hund's-rule coupling between itinerant $e_{g}$ electrons with $\boldsymbol{\sigma}_{i}$ spin and localized $t_{2 g}$ moments $\boldsymbol{S}_{i}$

Recently this simple DE scenario has been reexamined from the viewpoint of whether model (11) quantitatively describes thermodynamics in a typical material $\mathrm{La}_{1-x} \mathrm{Sr}_{x} \mathrm{MnO}_{3}$ (LSMO), or whether another mechanism is necessary. Based on mean-field (MF) arguments, Millis et al 4 ) claimed that the DE mechanism alone is insufficient to reproduce experiments. In particular, their estimate for the critical temperature $T_{\mathrm{c}}$ is one order of magnitude higher than experimental results. On the other hand, the validity of the DE scenario has peen claimed by dynamical mean-field (DMF) studies. Magnetism as well as transport and optical properties in the LSMO compound is well reproduced by model (1).

The DMF method includes dynamical fluctuations which were completely neglected in the MF study. DMF results suggest the importance of the fluctuations in this itinerant system. At the same time, the DMF method is not sufficient on its own since it takes into account only local fluctuations. In order to settle the controversy of whether the DE mechanism alone explains the thermodynamics of LSMO, it is necessary to make calculations which include all fluctuation effects.

A numerical study is one of the promising candidates for including the fluctuation effects appropriately. Many surveys based on the Monte Carlo (MC) method have been performed. However, their results have not yet converged mainly due to finite-size effects. Table I summarizes the values of $T_{\mathrm{c}}$ from the MC studies as well as by other approximations. MC calculations are performed for the system on finite-size clusters. In order to discuss critical phenomena, it is necessary to apply finite-size scaling analysis. However, such a systematic study has not been performed thus far since it has been difficult to study large-size clusters in three dimensions (3D) due to the rapid increase of the cpu time with the system size.

In this work we investigate the ferromagnetic transition in model (11) by finite-size scaling analysis of MC results. A new MC algorithm 11 is applied to reduce the cpu time markedly, which enables us to study larger clusters than ever. Our results elucidate effects of thermal and spatial fluctuations quantitatively. Our estimate for $T_{\mathrm{c}}$ agrees with those for LSMO in the ferromagnetic metal regime when parameters are chosen appropriately. Critical exponents are also examined in comparison with experimental results.

Model (1i) is studied with a classical treatment of the localized moments $\boldsymbol{S}$, as in other surveys listed in Table I. Then the fermion Hamiltonian defined for a given configuration of $\left\{\boldsymbol{S}_{i}\right\}$ becomes quadratic in the fermion operators and can be diagonalized with the matrix size proportional to the system size. Configurations of $\left\{\boldsymbol{S}_{i}\right\}$ are stochastically sampled in a MC fashion. The bottleneck of the standard MC technique $\mathcal{J}^{\text {J }}$ is the cost of the cpu time required to diagonalize the fermion Hamiltonian in each $\mathrm{MC}$ sample. The cost of cpu time is of the order of $N^{3}$ where $N$ is the system size. We apply a new algorithm 11 which replaces the exact diagonalization by a moment expansion of the density of states.12, 13 Calculations of the moments can be easily performed on parallel computers. The cpu time is reduced to the order of $\left(N^{2} \log N\right) / N_{\mathrm{PE}}$ where $N_{\mathrm{PE}}$ is the number of nodes in the parallel calculation. For details of the method, read- 
ers are referred to ref. 11 .

Table I. The critical temperature $T_{\mathrm{c}}$ of ferromagnetic transition in the DE model (1) estimated by various methods. (MF: meanfield approximation, HTE: high-temperature expansion, DMF: dynamical mean-field theory, MC: Monte Carlo calculation) The results are for $x=0.5$ and $J_{\mathrm{H}}=\infty$ except for noted cases. Errors in the last digit are shown in parentheses. $W$ is the half bandwidth of noninteracting electrons. The third column is $T_{\mathrm{C}}$ when $W$ is taken as $1 \mathrm{eV}$. The experimental result (EXP) is also denoted for comparison. See text for details.

\begin{tabular}{|c|c|c|c|}
\hline method & $\mathrm{T}_{\mathrm{c}} / W$ & $\mathrm{~T}_{\mathrm{C}}(\mathrm{K})$ & Ref. \\
\hline $\mathrm{MF}$ & $0.3^{\mathrm{a}}$ & $3500^{\mathrm{a}}$ & 4) \\
\hline HTE & 0.027 & 310 & 5) \\
\hline $\mathrm{DMF}$ & $\begin{array}{l}0.0809(3) \\
0.0714(1)^{b}\end{array}$ & $\begin{array}{l}942(4) \\
831(1)^{b}\end{array}$ & (6) \\
\hline $\begin{array}{l}\mathrm{MC} \\
\mathrm{MC} \\
\mathrm{MC}\end{array}$ & $\begin{array}{l}0.018(1) \\
0.0313(8) \\
0.0208\end{array}$ & $\begin{array}{l}210(10) \\
364(9) \\
242\end{array}$ & 盲) \\
\hline $\mathrm{MC}$ & $\begin{array}{l}0.037(3)^{\mathrm{c}} \\
0.0362(8)^{\mathrm{d}} \\
0.022(3)^{\mathrm{b}, \mathrm{c}} \\
0.0210(7)^{\mathrm{b}, \mathrm{d}}\end{array}$ & $\begin{array}{l}430(40)^{\mathrm{c}} \\
422(9)^{\mathrm{d}} \\
260(40)^{\mathrm{b}, \mathrm{c}} \\
245(8)^{\mathrm{b}, \mathrm{d}}\end{array}$ & $\begin{array}{c}\text { present } \\
\text { work }\end{array}$ \\
\hline EXP & & $369^{\mathrm{e}}$ & 10) \\
\hline
\end{tabular}

a. $x=0.3$ and $J_{\mathrm{H}}=\infty, \quad$ b. $x=0.5$ and $J_{\mathrm{H}}=4 W$

c. without assumption for the exponent $\beta$

d. with assumption $\beta=0.365$

e. $x=0.3$

We consider the hopping $t_{i j}=t$ only between nearestneighbor sites on a $3 \mathrm{D}$ cubic lattice. In the following we study the ferromagnetic transition for two parameters, $J_{\mathrm{H}}=\infty$ and $4 W$, where $W$ is the half bandwidth of noninteracting electrons ( $W=6 t$ in $3 \mathrm{D})$. For the case of $J_{\mathrm{H}}=\infty$, components of conduction electrons with spins antiparallel to localized moments are projected out, and therefore model (11) simply takes the spinless form of $\mathcal{H}=-\tilde{t} \sum_{i j}\left(c_{i}^{\dagger} c_{j}+\right.$ h.c. $)$, where $\left.\tilde{t}=t\left[\cos \left(\theta_{i} / 2\right) \cos \left(\theta_{j} / 2\right)+\sin \left(\theta_{i} / 2\right) \sin \left(\theta_{j} / 2\right) e^{\mathrm{i}\left(\phi_{j}-\phi_{i}\right)}\right] .14\right)$ The matrix dimension of the Hamiltonian is halved and the cpu time is reduced. Moreover, the moment expansion converges rapidly due to the simple band structure. In the following calculations, we perform the moment expansion up to the order of $M=20$ for $J_{\mathrm{H}}=\infty$ and $M=40$ for $J_{\mathrm{H}}=4 W$ in order to ensure sufficient precision for MC updates.11) For both values of $J_{\mathrm{H}}$, we control the chemical potential so that the electron density $x=\left\langle\sum_{i \sigma} c_{i \sigma}^{\dagger} c_{j \sigma}\right\rangle$ becomes 0.5 . Here, the bracket denotes the thermal average for the grand canonical ensemble. The critical temperature $T_{\mathrm{c}}$ in model (1) is known to have a maximum at $x \simeq 0.5$. A closed-shell condition is necessary to obtain full polarization in the ground state $(T=0)$. We chose the system size $N=N_{x} \times N_{y} \times N_{z}$ as $4 \times 4 \times 4,6 \times 6 \times 4,6 \times 6 \times 8$ and $8 \times 8 \times 8$ under the periodic boundary conditions for the $x$ and $y$ directions and the antiperiodic boundary condition for the $z$ direction. We have typically run 10,000 MC samplings for measurements after $1,000 \mathrm{MC}$ steps for thermalization. Measurement is divided into five bins to estimate the statistical error by the variance among the bins.

We determine the critical temperature $T_{\mathrm{c}}$ by two quan- tities. One is the Binder parameter 15) defined by

$$
g=1-\left\langle\boldsymbol{q}^{4}\right\rangle / 3\left\langle\boldsymbol{q}^{2}\right\rangle^{2},
$$

where $\boldsymbol{q}$ is the order parameter which is taken as $\sum_{i} \boldsymbol{S}_{i}$ in this case. For $T>T_{\mathrm{c}}\left(T<T_{\mathrm{c}}\right)$, the Binder parameter decreases (increases) as the system size $N$ increases. Thus $T_{\mathrm{c}}$ is determined by a crossing point of $g$ for various $N$. The other quantity is the magnetization defined by

$$
m=[S(\boldsymbol{k}=0) / N]^{1 / 2},
$$

where the spin structure factor $S(\boldsymbol{k})$ is given by

$$
S(\boldsymbol{k})=\sum_{i j}\left\langle\boldsymbol{S}_{i} \boldsymbol{S}_{j}\right\rangle e^{\mathrm{i} \boldsymbol{k} \cdot \boldsymbol{r}_{i j}} / N .
$$

The thermodynamic limit of $m$ is obtained from the system-size extrapolation of $S(0)$.

The case of $J_{\mathrm{H}}=\infty$ is discussed first. Figure 1 shows the Binder parameter for this case. The Binder parameters for different system sizes cross at $T \sim 0.035 \mathrm{~W}$ although it is difficult to determine $T_{\mathrm{c}}$ precisely due to the statistical error bars.

We next study the magnetization. Figure 2(a) displays the system-size extrapolation of $S(0) / N$. The data for $T \leq 0.035 W$ scale well with $N^{-2 / 3}$, and are extrapolated to finite values in the limit of $N=\infty$. This scaling is consistent,with $\boldsymbol{k}^{2}$-dependence of the energy cutoff for magnons.16) The extrapolated values are summarized as the magnetization in Fig. 2(b). The onset of the finite magnetization $m$ agrees well with the crossing point of the Binder parameter in Fig. 1. The estimate for $T_{\mathrm{c}}$ is $T_{\mathrm{c}} \simeq(0.037 \pm 0.003) W$.

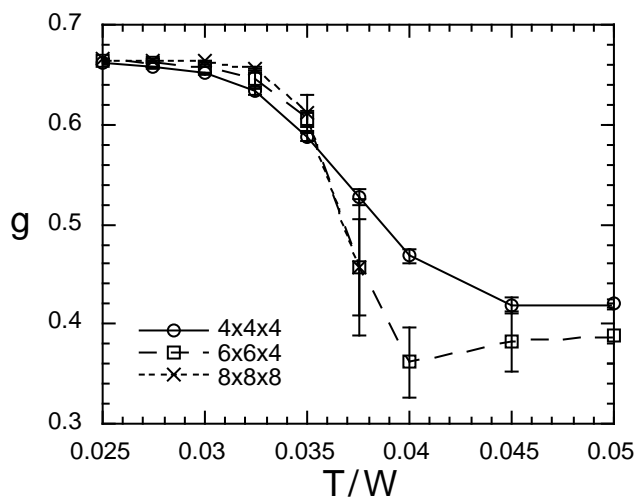

Fig. 1. Binder parameter for $J_{\mathrm{H}}=\infty$. The lines are guides to the eye.

To determine the value of $T_{\mathrm{c}}$ precisely, we fit the magnetization data by assuming the scaling law of $m \propto$ $\left(T_{\mathrm{c}}-T\right)^{\beta}$. The value of the exponent $\beta$ has not been determined thus far. At present, our data are insufficient to make estimates for both $T_{\mathrm{c}}$ and $\beta$ simultaneously within the desired accuracy. Instead we assume the exponent in 3D Heisenberg models $\beta=0.365,17$ ) and determine the value of $T_{\mathrm{c}}$. The data are well fitted, as shown in Fig. 2 (b). The fit gives $T_{\mathrm{c}}=(0.0362 \pm 0.0008) W$.

The assumption of $\beta$ in the universality class of $3 \mathrm{D}$ Heisenberg models is tested by finite-size scaling analysis. The scaling hypothesis gives the scaling relation for the 
spin structure factor (4) in the form

$$
S(0)=L^{2-\eta} f\left(L\left|T-T_{\mathrm{c}}\right|^{\nu}\right),
$$

where $L=N^{1 / 3}$ and $f$ is the scaling function. The critical exponents are predicted to be $\eta=0.034$ and $\nu=$ 0.705 in this universality class.17) We plot $S(0) / L^{2-0.034}$ as a function of $L\left|T-T_{\mathrm{c}}\right|^{0.705}$ with $T_{\mathrm{c}}=0.0362 \mathrm{~W}$ in Fig. 3 . The data appear to follow a universal function (there are two branches for $T>T_{\mathrm{c}}$ and $T<T_{\mathrm{c}}$ ) in this temperature range. This is consistent with the assumption of $\beta=0.365$ in the fit in Fig. 2(b). Later we will discuss the critical exponents in comparison with the experimental results.
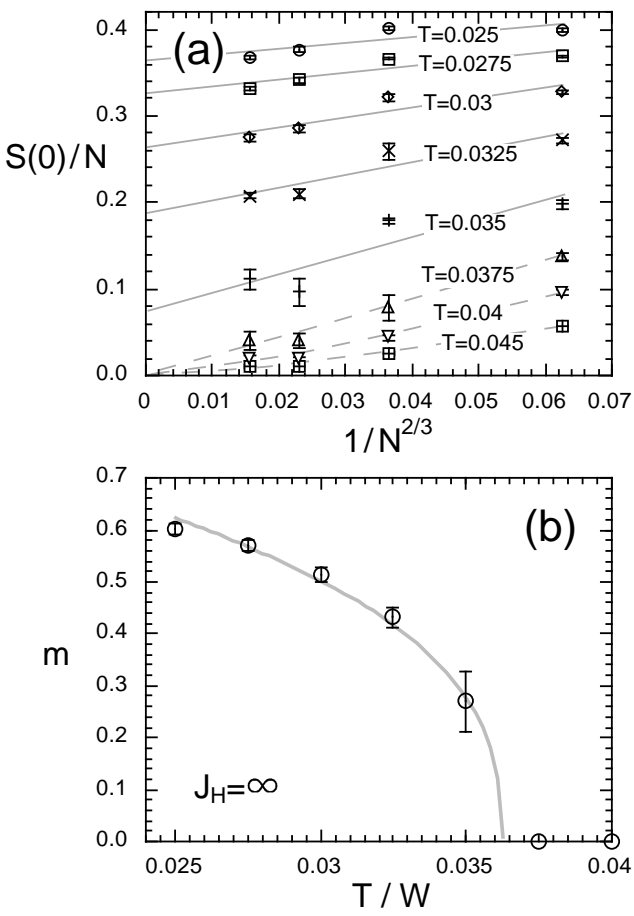

Fig. 2. (a) System-size extrapolation of the spin structure factor and (b) magnetization in the thermodynamic limit for $J_{\mathrm{H}}=\infty$. The curve in the figure is the least-squares-fit to $m \propto\left(T-T_{\mathrm{c}}\right)^{\beta}$. See text for details.

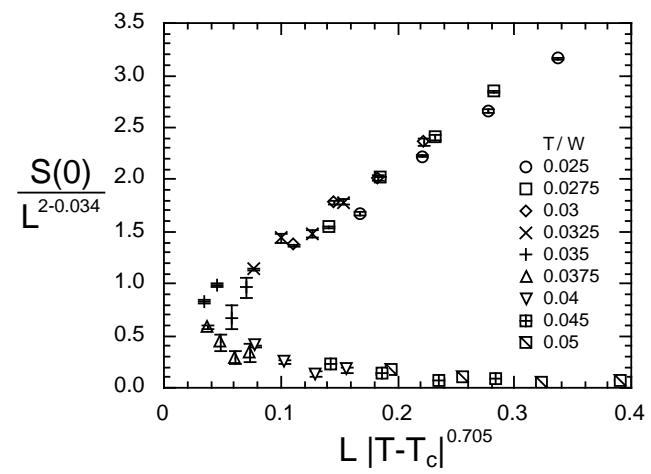

Fig. 3. Finite-size scaling for $J_{\mathrm{H}}=\infty$.

We now turn to the case of $J_{\mathrm{H}}=4 W$. As in the case of $J_{\mathrm{H}}=\infty$, an estimate for $T_{\mathrm{c}}$ is obtained more accurately by the magnetization measurement. Figure 4 shows (a) the system-size extrapolation of $S(0) / N$ and (b) the magnetization in the thermodynamic limit. We have $T_{\mathrm{c}} \simeq(0.022 \pm 0.003) W$ from Fig. $4(\mathrm{~b})$. If we assume the scaling $m \propto\left(T_{\mathrm{c}}-T\right)^{\beta}$ with the 3D Heisenberg exponent $\beta=0.365$ as in Fig. 2(b), the fit for $T \leq 0.02 W$ gives $T_{\mathrm{c}}=(0.0210 \pm 0.0007) W$ in this case.
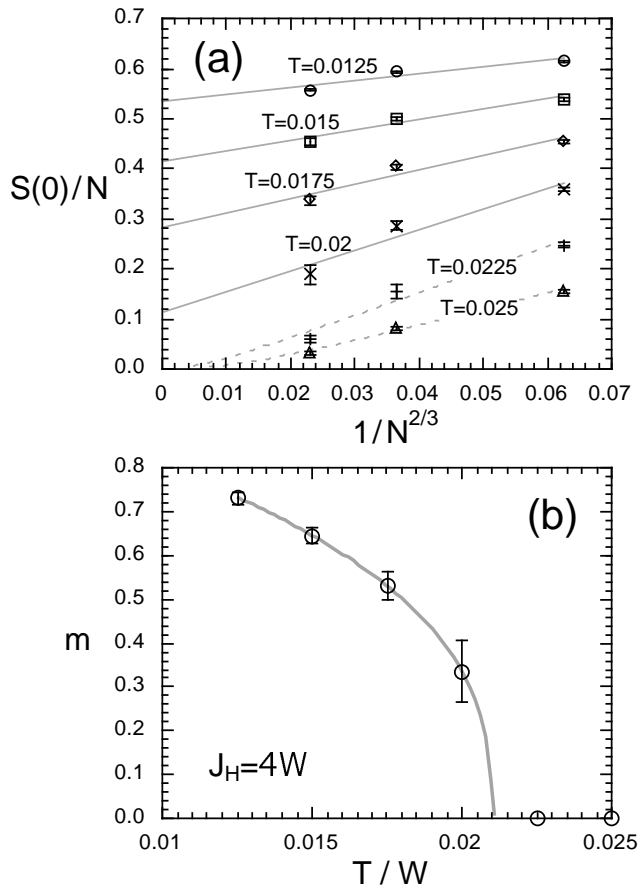

Fig. 4. (a) System-size extrapolation of the spin structure factor and (b) magnetization in the thermodynamic limit for $J_{\mathrm{H}}=4 \mathrm{~W}$. The curve in the figure is the least-squares-fit to $m \propto\left(T-T_{\mathrm{c}}\right)^{\beta}$. See text for details.

We compare the results of $T_{\mathrm{c}}$ with the other theoretical estimates listed in Table I. Our results are one order of magnitude lower than the MF estimates. This elucidates the importance of the strong fluctuations in this system. The MF approximation neglects all the fluctuations, i.e., the thermal and spatial ones. The DMF theory gives an intermediate approximation in the sense that it includes only the thermal fluctuation and neglects the spatial one. Comparison among MF, DMF and our $\mathrm{MC}$ results clarifies the fluctuation effects quantitatively: the thermal fluctuation significantly suppresses $T_{\mathrm{c}}$ from $0.3 W$ to $\sim 0.08 W$, and moreover the spatial fluctuation reduces $T_{\mathrm{c}}$ by more than half from $\sim 0.08 \mathrm{~W}$ to $\sim 0.04 \mathrm{~W}$ in the case of $J_{\mathrm{H}}=\infty$. Both fluctuations are important and cannot be neglected in a discussion of the phase transition.

The previous MC results listed in Table I deviate considerably from our results. These deviations arise from the finite-size effects and the method used to determine the transition temperature. $T_{\mathrm{c}}$ has been estimated by spin correlation between the farthest sites, $\$$ the second derivative of the magnetization 8 ) and the scaling fit for the data of a finite-size cluster. In order to discuss the phase transition in the strict sense, these methods are uncontrolled in the absence of system-size extrapolation.

The values of $T_{\mathrm{c}}$ are compared with the experimental result in Table I. Here, we take the half bandwidth $W$ to be $1 \mathrm{eV}$, which is typical for $3 d$ transition metal ox- 
ides. The bandwidth $-W=1 \mathrm{eV}$ is also consistent with band calculations. $18,29,20$ Thus our results, $T_{\mathrm{c}} \simeq 430 \mathrm{~K}$ for $J_{\mathrm{H}}=\infty$ and $T_{\mathrm{c}} \simeq 260 \mathrm{~K}$ for $J_{\mathrm{H}}=4 W$, agree well with the experimental values of $T_{\mathrm{c}}$ in the ferromagnetic metal regime, for instance, $T_{\mathrm{c}}=369 \mathrm{~K}$ at $x=0.3$. More quantitative agreement will be obtained by tuning the value of $J_{\mathrm{H}} / W$ and the doping concentration $x$. We conclude that once the fluctuation effects are fully taken into account, the DE mechanism gives a quantitative description of $T_{\mathrm{c}}$ in LSMO as a major origin.

Our results strongly support a view of LSMO in the ferromagnetic metal regime around $x=0.3$ as a canonical DE system, which has been pointed out by the DMF studies.2) Note that the compound is far from an antiferromagnetic/ferromagnetic insulator at $x \lesssim 0.1521$ and an $A$-type antiferromagnetic metal at $x \gtrsim 0.5$.22 These instabilities are beyond the scope of model (1). Detailed comparison between experiments involving this compound and theories in model (1) provides a good starting point to understanding not only the above instabilities but physical properties in other related materials. MC study of other physical properties in $3 \mathrm{D}$ is a subject for further study.

In our analysis, the universality class of 3D Heisenberg models is assumed. We also analyze the data by assuming the universality class of the mean-field theory, i.e., $\beta=\nu=0.5$ and $\eta=0$. In this case, the finite-size scaling plot (5) does not show good convergence compared to the data in Fig. 3. Our data fit better with the 3D Heisenberg exponents than do the mean-field ones. However, the present data are not sufficiently precise to either confirm the universality class of the DE model as that of the 3D Heisenberg model, or to identify it as a new universality class with similar exponents.

Concerning the experiments, values of the critical exponents for LSMO remain controversial. Estimates for $\beta$ are scattered in the range of $0.3 \lesssim \beta \lesssim 0.5 .23 .24 .25,26.27$ A recent study claims that the estimate for the critical exponent may depend on the range of the critical region assumed for fitting:28) $\beta \simeq 0.5$ is obtained for the relatively narrow critical region $\left(T_{\mathrm{c}}-T\right) / T_{\mathrm{c}} \lesssim 0.1$ while $\beta \simeq 0.3$ is found for the relatively wide critical region $\left(T_{\mathrm{c}}-T\right) / T_{\mathrm{c}} \gtrsim 0.2$. In our calculation, data of the magnetization are fitted over the wide range of $\left(T_{\mathrm{c}}-T\right) / T_{\mathrm{c}} \lesssim 0.4$. Considering the experimental situation, we cannot exclude the possibility that the results for the exponents in the DE model may be modified when the system further approaches to the critical point. Further investigation is required to determine the value of the exponents with precision.

To summarize, we have investigated ferromagnetic transition in the three-dimensional double-exchange model by Monte Carlo calculations. The new algorithm based on the moment expansion method has been applied to study large clusters. The critical temperature is determined precisely by finite-size scaling analysis. Comparison with other theoretical surveys quantitatively elucidates the importance of thermal and spatial fluctuations in this itinerant system. The critical temperature estimated with appropriate parameters agrees well with experiments in the ferromagnetic metal regime of
( $\mathrm{La}, \mathrm{Sr}) \mathrm{MnO}_{3}$. The double-exchange mechanism alone is sufficient to reproduce the ferromagnetic transition temperature in this material. The critical exponent in our analysis is consistent with experimental trends although more detailed study is necessary both theoretically and experimentally.

The authors thank H. Nakata for helpful support in developing parallel-processing systems. The computations have been performed mainly using the facilities in the AOYAMA+ project (http://www.phys.aoyama.ac.jp/ aoyama + ). This work is supported by "a Grant-in-Aid from the Ministry of Education, Science, Sports and Culture".

[1] A. P. Ramirez: J. Phys.: Condens. Matter 9 (1997) 8171, and references therein.

[2] N. Furukawa: in Physics of Manganites, eds. T. Kaplan and S. Mahanti (Plenum Publishing, New York, 1999), and references therein.

[3] C. Zener: Phys. Rev. 82 (1951) 403.

[4] A. J. Millis, P. B. Littlewood and B. I. Shraiman: Phys. Rev. Lett. 74 (1995) 5144.

[5] H. Röder, R. R. P. Singh and J. Zang: Phys. Rev. B 56 (1997) 5084 .

[6] N. Furukawa: J. Phys. Soc. Jpn. 64 (1995) 2754. The values of $T_{\mathrm{c}}$ in Table I are calculated for the density of states in 3D in the thermodynamic limit.

[7] S. Yunoki, J. Hu, A. Malvezzi, A. Moreo, N. Furukawa and E. Dagotto: Phys. Rev. Lett. 80 (1998) 845.

[8] M. J. Calderón and L. Brey: Phys. Rev. B 58 (1998) 3286.

[9] H. Yi , N. H. Hur and J. Yu: Phys. Rev. B 61 (2000) 9501.

[10] Y. Tokura, A. Urushibara, Y. Moritomo, T. Arima, A. Asamitsu, G. Kido and N. Furukawa: J. Phys. Soc. Jpn. 63 (1994) 3931.

[11] Y. Motome and N. Furukawa: J. Phys. Soc. Jpn. 68 (1999) 3853.

[12] L. W. Wang: Phys. Rev. B 49 (1994) 10154.

[13] R. N. Silver and H. Röder: Int. J. Mod. Phys. C 5 (1994) 735.

[14] P. W. Anderson and H. Hasegawa: Phys. Rev. 100 (1955) 675.

[15] K. Binder: Z. Phys. B 43 (1981) 119.

[16] N. Furukawa: J. Phys. Soc. Jpn. 65 (1996) 1174.

[17] J. C. Le Guillou and J. Zinn-Justin: Phys. Rev. Lett. 39 (1977) 95.

[18] N. Hamada, H. Sawada and K. Terakura: in Proc. 17th Taniguchi International Conference, eds. A. Fujimori and Y. Tokura (Springer-Verlag, Berlin, 1995).

[19] W. E. Pickett and D. J. Singh: Phys. Rev. B 55 (1997) 8642.

[20] D. A. Papaconstantopoulos and W. E. Pickett: Phys. Rev. B 57 (1998) 12751.

[21] A. Urushibara, Y. Moritomo, T. Arima, A. Asamitsu, G. Kido and Y. Tokura: Phys. Rev. B 51 (1995) 14103.

[22] T. Akimoto, Y. Maruyama, Y. Moritomo and A. Nakamura: Phys. Rev. B 57 (1998) 5594.

[23] R. H. Heffner, L. P. Le, M. F. Hundley, J. J. Neumeier, G. M. Luke, K. Kojima, B. Naomichi, Y. J. Uemura, D. E. MacLaughlin and S-W. Cheong: Phys. Rev. Lett. 77 (1996) 1869.

[24] M. C. Martin, G. Shirane, Y. Endoh, K. Hirota, Y. Moritomo and Y. Tokura: Phys. Rev. B 53 (1996) 14285.

[25] S. E. Lofland, V. Ray, P. H. Kim, S. M. Bhagat, M. A. Manheimer and S. D. Tyagi: Phys. Rev. B 55 (1997) 2749.

[26] K. Ghosh, C. J. Lobb, R. L. Greene, S. G. Karabashev, D. A. Shulyatev, A. A. Arsenov and Y. Mukovskii: Phys. Rev. Lett 81 (1998) 4740.

[27] L. Vasiliu-Doloc, J. W. Lynn, Y. M. Mukovskii, A. A. Arsenov and D. A. Shulyatev: J. Appl. Phys. 83 (1998) 7342.

[28] A. Schwartz, M. Scheffler and S. M. Anlage: Phys. Rev. B 61 (2000) 870. Note that this experiment is for $x=0.2$. 